THURSDAY, JULY 9,1874

\section{THE COMET}

70 those who are familiar with the triumphs which that most wonderful of modern instruments of research-the spectroscope-has achieved, the short time during which it has been at work will be most forcibly recalled by a reference to the circumstance that the comet which is now, astronomically speaking, a magnificent object in the northern sky, is the first one of any considerable brilliancy which has shown itself since the spectroscope has been adapted to the telescope.

The truly splendid comets which delighted us during the autumn of 1858 , and for a brief space in the summer of $186 \mathrm{r}$, made their appearance, in fact, during what we may term the pre-spectroscopic age; for, however little to the credit of modern science it might have been that the spectroscope was no employed in their investigation, the fact remains that they were allowed to pass away mere telescopic objects, and that two opportunities were thus lost such as, perhaps, may not offer themselves again to the present generation of men.

I propose, in the present paper, to state some poinis of inquiry regarding comets in which the spectroscope may help us, with a view of showing how much closer is our grip of celestial phenomena when physical astronomy, in its widest sense, is superadded to the older astronomy, and to indicate the numerous gains to knowledge which may be hoped for if adequate telescopes, properly armed with spectroscopes, are employed both here and in the southern hemisphere upon the present visitor.

Omitting all reference to the paths of comets round the sun, with which mechanical astronomy has to do, there are perhaps but few points in which the spectroscope cannot help us; somewhat unfortunately, however, there is one in which it appears powerless, and that precisely one of the greatest difficulty in cometary theory. I allude to the apparent sweep of the tail round the sun when the comet is at its perihelion point, which has suggested to Faye a theory of a repulsive force due to solar heat, and which perhaps is one of the most mysterious phenomena which we witness in the skies. Leaving this aside, however, there are many questions relating to what Sir John Herschel terms their "interior economy," in which, undoubtedly, the guesses of telescopic observers may be turned into hard, detailed fact.

Let us briefly refer to some of these points.

Generally speaking, as a comet approaches the sun it gets brighter and its tail lengthens, whether the nucleus is intensely stellar, as in the present case, or not ; in some cases a violent action may be observed ; aigrettes, or jets, make their appearance; and the nucleus, or head, is surrounded, or partly surrounded, by envelopes or shells, very obvious and with marked boundaries, and these are visible in some cases at the commencement of the tail.

Now, of course, if any or all of these luminous phenomena were due to the reflection of sunlight by masses of whatever kind not luminous in themselves, then the spectrum would be the same from all, differing only in intensity, and the spectrum would be the true solar spectrum if there

VOL, $\mathrm{x},-$ No. 245 were light enough, and a dim continuous spectrum if the part of the comet under examination were dim.

If, on the other hand, the masses were self-luminous and consisted of vapours not too dense, then we should get a characteristic spectrum proving first the existence of vapours driven into incandescence; and secondly, if the observations went far enough, the precise quality or nature of the vapour would be determined for us by the spectroscope. Thanks to the labours of Donati, Huggins, Secchi, Wolf, Rayet, Vogel, and others, the brightest portions of the comets which have appeared since 1864 have been examined with the undoubted result that they consist, in part at least, of not very dense incandescent vapour. I say in part, because in some cases the continuous spectrum, which may denote dense vapours, or perhaps vapours of relatively greater molecular complication, or again even glowing solid substances, has been so strong as almost entirely to mask the bright lines or bands by means of which the presence of the rarer or simpler vapours is determined.

Nor is this all. Not only have lines been seen, but their positions have been determined with some degree of accuracy, although it must be pointed out that the opinions of authorities do not coincide as to the actual materials indicated or as to the interpretation to be put upon the observations. This is not to be wondered at, considering the amazing delicacy of the research and the few opportunities there have yet been of making perfectly satisfactory determinations.

The most searching criticism of the results hitherto obtained appeared some little time ago in Poggendorff's Anraten from the pen of Dr. Vogel (NATURE, vol. ix. p. 193), and it will be well to briefly glance at some points which result from his inquiry. Donati, in the first observations of this nature made in 1864, determined the existence of three bright bands, but made no attempt to determine the substance from which the light proceeded. Huggins in 1866 made the first attempt in this direction, and came to the conclusion that, like the nebulæ, the comets might be composed of nitrogen, as in the spectrum of the comet visible in that year there was a single line which nearly, if not quite, coincided with one of the brightest lines of that element. In 1868, however, the idea of nitrogen comets was abolished, as the idea of nitrogen nebulæ has been since; and the three bands, which were again observed in the comets visible in that year, were found to coincide with those of olefiant gas. Hence it was suggested by Huggins that they consisted of carbon vapour. He writes :- "The great fixity of carbon seems indeed to raise some difficulty in the way of accepting the apparently obvious inference of these prismatic observations. Some comets have approached the sun sufficiently near to acquire a temperature high enough to convert carbon into vapour. Indeed, for these comets a body of great fixity seems to be necessary. . . If the substance of the comet be taken to be pure carbon, it would appear that the nucleus had been condensed from the gaseous state in which it existed at some former period. . . . If we were to conceive the comet to consist of a compound of carbon and hydrogen . . other difficulties would arise in connection with the decomposition we must then suppose to take place . . ."

It is clear that Mr. Huggins' opinion is that a comet 
consists of carbon; that the vapour is carbon vapour driven into incandescence by a temperature high enough to volatilise carbon, and not the vapour of a volatile hydrocarbon.

Such is not M. Vogel's view, and I confess it is not mine. After giving details of the observations of the nine comets examined between 1864 and I 87 r, M. Vogel thus analyses them :--

"Of these nine comets, there is only one ( 1870 ) for which we have no observations as to the position of the bright bands. Of the remaining eight, the spectra of five ( 1,2 , 4,7 , and 9) have shown no agreement with the hydrocarbon spectrum. As regards the Comet II. I 867 the supposition is offered that its spectrum was similar to the spectrum named; as to Encke's Comet III. 187I, it remains uncertain in which class it is to be reckoned (Huggins' observations being at variance with those of Young and myself). There remains only the Comet II. I868, for which Huggins' and Secchi's observations assert a probability of coincidence of the lines in its spectrum with those in the spectra of volatile hydrocarbons.

"It thus appears a somewhat questionable view, that the comets consist of such matter; and we should, I think, content ourselves with the deduction that a portion of the light emitted by the comet is its own light, and very probably from glowing gas."

Hence, then, the whole question of the true material of which that part of the comet consists, the spectrum of which has been already observed, must be acknowledged as being still sub judice: and this is a matter of the first order of importance, on which the present comet may throw much light.

But one of the most hopeful points is this: the comets up to the present time have been either so small or so distant that the record of aigrettes or envelopes on the spectrum has not been cietermined; nay, the comets might have been deprived of those appendages, hence the statement concerning the spectrum is a very general one; there has been no sufficient opportunity of localising the spectrumngiving region or regions.

What a glorious harvest will be reaped should the jets appear as decided as in the comet of r 861 , or in Halley's comet at its return in 1835 ; "jets, as it were, of flame, or rather of luminous smoke, like a gas fan-light," which, as described by Sir John Herschel, "varied from day to day as if waving backwards and forwards, as if they were thrown out of particular parts of the internal nucleus or kernel, which shifted round, or to and fro, by their recoil, like a squib not held fast."

Or again, suppose the system of concentric envelopes $i_{S}$ developed to the same extent as in Donati's comet, in which the action at all points of the nucleus, to follow Sir Joh n Herschel's reasoning, was probably more general, a result due to a more uniform chemical constitution.

Hence the comet may leave us a rich inheritance in the shape of "spectrum of jets," or "spectrum of envelopes;" and from what I have already seen dimly (for such observations are beyond my instrumental power), the former is the more probable, and in the nucleus we may have the equivalent of the sun, or the carbon pole of an electric lamp, with a continuous spectrum, and in the jets phenomena identical with those presented by solar storms, or the electric arc, that is, lines of various lengths indicating various vapours, shooting out or extending to various distances according to their volatilities, or vapour densities.
We seem, indeed, to have got a true physical approximation to this state of things in the comet of 1868 , for $\mathrm{Mr}$. Huggins observed that while some of the lines thinned out as one sees them do in the ordinary spark by using a lens, quite independently of the general visibility of the vapour, others did not so thin out, but retained their breadtil till they disappeared altogether.

The extent to which this action will go on will obviously depend upon two things, first the temperature and secondly the materials of the comet; and this raises an important question, which perhaps is easier of solution than the determination of the materials ejected, should that phenomenon be spectroscopically recognisable.

I have already communicated to NATURE the fact that to me the continuous spectrum of the nucleus appears deficient in blue rays. The effect of this upon the colour of the nucleus would be to give it a yellowish tinge like that of a candle flame, and for the same reason.

Dr. Vogel, in the paper to which I have already referred, deals with this question of colour, stating that:-

"Dr. Zenker arrives at the conclusion that there must be water-vapour in the comets ; since they have, according to Schmidt, a yellowish-red colour, and the sun's rays, when they pass through a considerable thickness of aqueous vapour, are coloured thus. But apart from the consideration that sunlight has a yellowish-red colour on passing through other vapours as well as aqueous, I would remark that we must take the proper light of the comet, which appears from spectral analytic observations to be generally more intense than the reflected light, as determining its colour. According to the observations made, we should expect that the comet is, on the whole, of greenish or greenish-blue colour, since all the spectra consist, as we have seen, of two or three bands of light, of which one is in the yellow, the second and brightest in the green, and the weakest in the beginning of the blue. Of the (generally very faint) continuous spectrum, only the brightest part-yellow, green, and commencement of blue - is visible. The entire innage, therefore, even where the weak continuous spectrum appears, will seem of greenish colour. Colour-data have been furnished by other observers besides Schmidt ; and the head of the Comet I 8 II, c.g. had, according to Herschel, a greenish or bluish colour; the nucleus was slightly red. The colour of Halley's comet, at its return in 1825, was a bluish-green (Struve). Winnecke says of the comet of 1862 , "The colour of the neck appears to me yellowish; the coma has bluish light." "

It will be seen that these remarks are quite in accordance with the suggestion. Dr. Zenker attributes to absorption the effect which I ascribe to defective radiation, and if it should be determined that the spectrum of the nucleus is truly deficient in blue rays, then a great point will be gained, for its temperature must be low.

Angstıom, whose death the world of science is now de. ploring, lived to say that he conceded that different molecular arrangements of the same element might give us different spectra ; and Roscoe and Schuster have recently placed beyond all doubt that, besides the well-known high temperature spectra of sodium and potassium, there are other spectra appertaining to the vapour of these elements at a lower temperature.

Now these spectra are channelled-space spectra, that is similar in character to the spectrum which has already been observed in the case of comets; and if such spectra be obtained for all elements (and $I$ have already added to the list), if a comet be a body at a low temperature, it is 
such spectra as these that we shall see, and not line spectra. Further, in the case of compounds in which the molecules which give us these new spectra enter into combination, we may possibly dissociate them and observe their spectra at a much lower temperature than we can drive the higher molecular arrangement of the solid into vapour,

Such considerations as these derive additional interest and importance from the beautiful researches of Schiaparelli, which connect comets with meteorites.

Modern science acknowledges that comets are individual members of meteor swarms-not that meteors are comets' tails, as some think; this idea is, one may say, impossible to reconcile with facts-that one difference at any rate between a comet and a meteor is that one is self-luminous, the other is not till it arrives wit? in the limits of our atmosphere. If this be acknow1.uged, then to what is this difference to be ascribed? A possible cause is certainly a difference of chemical constitutiona difference between niaterials incandescent at a high temperature and materials incandescent at a low one. It is not necessary to stop to inquire how this temperature has been arrived at, but it is important to show that the question of temperature is one of the very first poinis to be attended to by those who can kring sufficiently powerful instruments to bear upon the present comet, and that the question of its actual chemical constitution is bound up with it.

But whatever be the temperature of the head there is another point which must not be lost sight of. Sir John Herschel writes concerning Halley's comct: "The bright smoke of the jets, however, never seem to be able to get far out towards the sun, but always to be driven back and forced into the tail, as if by the action of a violent wind rolling against them-always from the sun-so as to make it clear that this tail is neither more nor less than the accumulation of this sort of luminous vapour, darted off in the first instance towards the sun, as if it were something raised up, and as it were exploded by the sun's heat, out of the kernel, and then immediately and forcibly turned back and repelled from the sun." Here we have the question raised not only whether the envelopes consist of different materials, but whether the tail is not entirely or in part seif-luminous: the present comet may show that this point is not so satisfactorily settled as it is supposed to be in favour of reflected light.

Such then are briefly some of the questions at issue. It is to be hoped that our beautiful visitor will answer some of thern for us, and that when it leaves our northern skies the work may be carried on in the southern hemisphere.

J. NORMIAN LOCKYER

\section{THE CHANNEL TUNNEL}

WE fear there are still many who fail to see that any good can come of scientific research unless it has some well-defined "utilitarian" object in view. Even in this and in other comntries that are in the van of civilisation and in which education is comparatively wide-spread, the majority of mankind can appreciate a benefit only when it takes a concrete and tangible form. That love of knowledge for its own sake, that noble inquisitiveness which has been so fruitful in results during the last two hundred years, even yet belongs to comparatively few, who are still regarded by the many with a kind of im- patient pity as mere unpractical hobby-riders. Still the people who talk in this way are proud enough of the glory which their great men have shed upon their country, and would not willingly, we believe, part with it for money were this possible; and indeed how would this country appear among the nations were she deprived of the inestimable inheritance which her great sons have bequeathed to her in every department of intellectual activity? Happily, however, the race of those who decry single-eyed scientific research is getting sensibly smaller; and we firmly believe that as education improves and as higher education spreads, carrying with it the results of this same scientific research, it will disappear.

Still, a little consideration might show those who are ever ready to cry "what's the good?" that since all socalled "practical" schemes are concerned either with man's own body or with the surrounding universe, an essential part of the basis of any scheme is a thorough knowledge of the material on which it is proposed to work. Such a knowledge it has over and over again been shown is only to be attained by abstract scientific research, by investigation conducted as if the only end in view were a thorough knowledge of the subject in hand in all its scientific aspects and relations. Many instances could be given, and indeed are every day occurring, of the highest practical results unwittingly following from such investigations; and to the sceptic we could not recommend a better example of how indispensable is thorough scientific research as a basis for the useful arts than the results of the investigation into the geology of the Channel which Mr. Prestwich (the newly elected Oxford Professor of Geology) presented to the Institution of Civil Engineers last December, and which, with the subsequent discussion and maps, has just been published in a separate form. This study of the strata which underlie the Channel, and which seems to us an almost perfect example of close and careful reasoning on physical facts, is now brought forward to enlighten the projectors of a tunnel between England and France as to the nature of the material with which they will have to work; but $\mathrm{Mr}$. Prestwich distinctly states that the various formations are considered "irrespective of their relative merits in any other than a geological point of view."

Mr. Prestwich's plan is to discuss carefully all the strata whicin underlie the Channel, from the London clay down to the Palæozoic series, exhibiting distinctly their lithological characters, dimensions, range, and probable depth, and from these data deducing his conclusions as to the suitability of each formation for being pierced by a tunnel. The investigations of himself and others on which Mr. Prestwich's paper is founded were mostly undertaken from no practical point of view, and before a Channel tunnel was thought of. Mr. Prestwich, many will be glad to think-grateful, we hope, at the same time for this very practical result of pure scientific research-concludes that from a geological point of view it is quite practicable to construct a tinnel underneath the Channel, although to do so with safety it will be necessary to go very deep down. But an excellent idea of the results of the investigation will be obtained from the following clear summary with which Mr. Prestwich's paper concludes :--

"In the London clay there exists a perfectly impermeable bed of sufficient thickness, but nowhere between the two 